\section{Acute acquired comitant esotropia}

Acute acquired comitant esotropia describes a sudden onset of esotropia with diplopia and minimal refractive error. Although rare it requires recognition and thorough investigation due to its possible association with underlying central nervous system disorders.

Until recently it was generally agreed that as the esotropia was concomitant it was confirmation of the benign nature of the condition. Several reports, however, have shown this not to be the case. ${ }^{1-5}$ The emphasis of the reports is on careful examination of these patients, looking for any subtle signs of incomitancy or associated neurological signs or symptoms.

However, the rarity of underlying central nervous system disorders in patients with acute acquired comitant esotropia is also apparent when considering the relatively small number of patients presenting over an extended period in the above reports. This inevitably leads to controversy regarding the selection of these patients for neurological assessment. ${ }^{5,6}$ Many articles discuss the features suggestive of underlying pathology. 3,5,7,8,9

The paper by Lyons et al. ${ }^{10}$ in the current issue of Eye aims to provide some guidelines as to the necessity for neurological investigation based on the presenting clinical features of patients with acute acquired comitant esotropia. The particular value of this paper is that it is the first prospective study of this type of strabismus based on presentation of diplopia/closing one eye and clinical findings. Previous articles have reviewed patients or patient records with this condition $^{11,12}$ or reviewed patients with diagnosed pathology from acute acquired comitant esotropia. ${ }^{5,9}$ Of the 10 patients presenting with acute acquired comitant esotropia over a 3 year period in the Lyons et al. ${ }^{10}$ study, one patient was found with underlying pathology (cerebellar tumour). By studying the patients prospectively Lyons et al. ${ }^{10}$ are looking at typical and atypical features of all patients with acute acquired comitant esotropia, irrespective of the ultimate aetiology.

Timms and Taylor ${ }^{5}$ and Hoyt and Good ${ }^{3}$ discuss the variable time scale between the onset of this type of esotropia and the onset of the neurological signs and symptoms.
Consequently Lyons et al. ${ }^{10}$ reiterate the need for maintaining suspicion if there is a presence or absence of the typical clinical features.

The aetiology of acute acquired comitant esotropia was discussed by Burian and Miller ${ }^{13}$ in 1958. They divided this type of esotropia into three categories based on the clinical features and apparent aetiology:

Group 1 (Swan type): acute onset esotropia following occlusion which von Noorden ${ }^{14}$ refers to as the most common aetiology, although Legmann Simon et al. ${ }^{12}$ have not found this to be so.

Group 2 (Franceschetti type): refractive error is minimal hypermetropia with no accommodative element. Most authors following the Burian classification feel their patients fall into this group.

Group 3 (Bielschowsky type): acute acquired comitant esotropia associated with myopia.

Ellis and Pritchard ${ }^{15}$ in their attempt to classify acute acquired comitant esotropia include accommodative and cyclic esotropia. Macpherson et al. ${ }^{8}$ discuss decompensating esophoria and uncorrected refractive error and Legmann Simon et al. ${ }^{12}$ discuss the fact that simple refractive or accommodative esotropia are not included in the Burian classification.

Lyons et al. ${ }^{10}$ on studying the aetiology of their patients found 9 of the 10 patients to be hypermetropic, with decompensation of a preexisting esophoria or monofixation syndrome being the most common cause of this type of strabismus. The Burian classification may now be an oversimplification of acute acquired comitant esotropia as it does not make allowances for those with underlying pathology or the other aetiologies discussed above.

Whilst observing the clinical characteristics of the patients in the Lyons et al. ${ }^{10}$ article several features were noted. The presence of diplopia or closing of one eye was one of the criteria for inclusion in their study. The age range of the patients was 3.5 years to 24 years. This may indicate that this is the youngest age at which these features are distinguishable. Timms and Taylor ${ }^{5}$ discuss the problem of diagnosing this condition in the younger age group. Lang ${ }^{16}$ stresses the importance of the case history particularly in these younger patients. In association with the case history is any family history of refractive error or strabismus. Lyons et al. ${ }^{10}$ noted 4 of their 10 patients $(40 \%)$ to have
Lynn Baker

University Department of Ophthalmology and Orthoptics

O Floor

Royal Hallamshire Hospital Glossop Road Sheffield S10 2JF, UK 
a positive family history, one patient having a similar onset of acute esotropia. The patient in their study found to have a cerebellar tumour had no family history. However, lack of family history obviously does not stand alone as an identifying feature of those likely to have underlying pathology, as acknowledged by Lyons et al. ${ }^{10}$ and demonstrated by Anderson and Lubrow, ${ }^{1}$ whose patient had an astrocytoma with a family history of accommodative strabismus.

Nine of the 10 patients $(90 \%)$ were hypermetropic in the Lyons et al. ${ }^{10}$ study, which is obviously a very high proportion, the only patient with no refractive error being the one with the tumour. Again although this may seem significant Williams and Hoyt ${ }^{9}$ add a word of caution in their article as one of their patients responding to hypermetropic spectacles was later found to have a tumour and the response to spectacles delayed the eventual diagnosis.

Binocular function is assumed to be present prior to onset of the esotropia on the basis of its acquired nature. In the Lyons et al. ${ }^{10}$ article the patient with the tumour did not show potential binocularity, but neither did 3 other patients. Hoyt and Good ${ }^{3}$ discuss lack of binocularity following strabismus surgery as an indicator of underlying pathology. In contradiction to this Lyons et al. ${ }^{10}$ found the patient with the cerebellar tumour regained binocularity following strabismus surgery, the difference being that the tumour had been excised before the strabismus surgery. They suggest it may be length of time before diagnosis of the pathology that affects the restoration of binocularity as opposed to lack of binocularity being a diagnostic feature. The presence of abducting nystagmus is obviously an important feature in patients with acute acquired comitant esotropia when looking for subtle sixth nerve involvement. Although the importance of the presence of nystagmus is discussed in several articles ${ }^{3,5}$ it has been found in patients with diagnosed central nervous system disorders. The incidence of its occurrence in this type of esotropia generally is not discussed. Lyons et al. ${ }^{10}$ found 3 of their patients $(30 \%)$ to have abducting nystagmus, none of whom was the child with the cerebellar tumour.

In summary, the article by Lyons et al. ${ }^{10}$ is particularly valuable for two reasons. By considering the presenting aetiologies of their patients they allow us to widen our classification of this condition. In addition, although they could find no single clinical characteristic reliably indicating that the patient could have central nervous system disorders the article is still useful. By virtue of it being a prospective study it highlights several features, the presence or absence of these features being significant and suggesting that neurological assessment is required.

\section{References}

1. Anderson WD, Lubrow M. Astrocytoma of the corpus callosum presenting with acute comitant esotropia. Am J Ophthalmol 1970;69:594-8.

2. Jaafar MS, Collins MLZ, Rabinowitz AI. Cerebellar astrocytoma presenting as acquired comitant esotropia at age 18 and 27 months. In: Update on strabismus and pediatric ophthalmology. Boca Raton, FL: CRC Press, 1995:597-600.

3. Hoyt CS, Good WV. Acute onset concomitant esotropia: when is it a sign of serious neurological disease? Br J Ophthalmol 1995;79:498-501.

4. Simon JW, Waldman JB, Couture KC. Cerebellar astrocytoma manifesting as isolated comitant esotropia in childhood. Am J Ophthalmol 1996;121:584-6.

5. Timms C, Taylor D. Intracranial pathology in children presenting as concomitant strabismus. In: Update on strabismus and pediatric ophthalmology. Boca Raton, FL: CRC Press 1995:593-6.

6. Avilla C. Decompensated strabismus in adults. Am Orthopt J 1994;44:39-43.

7. DeYoung Smith M, Baker JD. Esotropia as the presenting sign of brain tumour. Am Orthopt J 1990;40:72-5.

8. Macpherson H, De Becker I, MacNeill JR. Beware: armed and dangerous - acquired non-accommodative esotropia. Am Orthopt J 1996;46:44-56.

9. Williams AS, Hoyt CS. Acute comitant esotropia in children with brain tumours. Arch Ophthalmol 1989;107:376-8.

10. Lyons CJ, Tiffin PAC, Oystreck OC. Acute acquired comitant esostropia: a prospective study. Eye 1999;13:617-20.

11. Clark AC, Nelson LB, Simon KW, Wagner R, Rubin SE. Acute acquired comitant esotropia. Br J Ophthalmol 1989;73:636-8.

12. Legmann Simon A, Borchert M. Etiology and prognosis of acute, late-onset esotropia. Ophthalmology 1997;104:1348-52.

13. Burian HM, Miller JE. Comitant convergent strabismus with acute onset. Am J Ophthalmol 1958;45:55-63.

14. von Noorden GK. Binocular vision and ocular motility, 5th edn. St Louis: CV Mosby, 1996:324-5.

15. Ellis GS, Pritchard CA. Childhood esotropia: the players. Am Orthopt J 1996;46:3-6.

16. Lang J. Normosensorial late convergent squint. In: Campos EC, editor. Fifth International Strabismological Association meeting, 1986:536-40. 